\title{
Experimental testing of the left periphery
}

Jose Sequeros-Valle

Department of Hispanic and Italian Studies, University of Illinois at Chicago, US

https://doi.org/10.36505/ExLing-2020/11/0045/000460

\begin{abstract}
This manuscript presents an empirical description of the left periphery based on the performance of speakers of Castilian Spanish in a corpus analysis, an acceptability judgment task, and a scripted production task. The picture drawn by the three studies look as follows: First, clitic-doubled left dislocations (CLLD) fulfil multiple discourse functions, but the construction is not completely free from discourse restrictions. Second, canonical utterances are also able to fulfil CLLD's discourse functions. Third, CLLD does not present distinctive intonational patterns depending on the discourse function. Fourth, there is partial evidence that focus fronting (FF) presents an intonational pattern different than that of CLLD. The concluding section of the manuscript calls from a new model of the left periphery.
\end{abstract}

Keywords: clitic-doubled left dislocation, focus fronting, left periphery, information structure, Spanish

\section{Background}

Much generative research in recent years has focused on the interaction of different linguistic subdomains, and how this interaction can help us develop a holistic model of the architecture of human language: Does phonology have its own interpretation system or it depends directly on morphology? Are semantics and discourse two different domains or two aspects of the same domain? Is discourse pre- or post-syntactic? One subdomain extensively studied is discourse, on its interface with syntax and phonology. The interface among these three domains is worth investigating because it has implications for the larger questions presented above, and for language at large.

In order to look into the discourse-syntax-phonology interface, I take the Spanish left periphery as a test case; namely clitic-doubled left dislocations (CLLD: A Pedro María lo ve en el parque, 'Pedro, Maria see him in the park'), focus fronting (FF: A Pedro ve Maria, 'Pedro Maria sees'), and their canonical counterpart (María ve a Pedro, 'Maria sees Pedro). In general terms, FF expresses contrastive focus and presents an emphatic phonology (low intermediate boundary tone, with an extended pitch range), while CLLD expresses topicality and presents a clitic pronoun doubling the fronted constituent.

Despite this general description, previous accounts disagree on the details. First, Bianchi and Frascarelli (2010) argue that CLLD can fulfil multiple discourse contexts. Further, these discourse functions cannot be fulfilled by

ExLing 2020: Proceedings of 11 th International Conference of Experimental Linguistics, 12-14 October 2020, Athens, Greece 
canonical utterances, and each interpretation corresponds to a unique intonation. In a second approach, López (2009) argues for a unique interpretation of each FF and CLLD, and these two discourse functions can be fulfilled by canonical utterances as well. Third, Rubio Alcalá (2014) argues for a completely interpretational freedom of all canonical and non-canonical utterances. Further, none of these authors have provided experimental evidence for their claims.

Based on the disagreements presented above, I propose four research questions for experimental testing: RQ1: Does Spanish CLLD fulfil multiple discourse contexts? RQ2: Do canonical utterances fulfil the same discourse contexts as Spanish CLLD? RQ3: Are different discourse-contexts marked via specific-pitch accent in Spanish? RQ4: Is contrastive focus marked via contrastive-stress in Spanish?

\section{Study 1: A corpus analysis}

The findings discussed in this section come from the Spanish section of the NOCANDO Corpus (Brunetti et al., 2011; available via https://parles.upf.edu/llocs/nocando/home). The corpus presents 32 potential instances of CLLD and FF, but some of them did not include the characteristics described above. Therefore, I only present the findings from the remaining 20 instances of CLLD, and no instances of FF.

These 20 remaining instances of CLLD were coded following the guidelines from Götze et al. (2007), which showed to fulfil multiple discourse contexts (e.g., given, subset, etc.). Additionally, a qualitative analysis of the corpus indicated that canonical utterances also fulfil the same six discourse functions found for CLLD. The same 20 instances of CLLD presented multiple pitchaccents on an analysis in Praat (e.g., $\mathrm{L}+\mathrm{H}^{*}, \mathrm{~L}^{*}+\mathrm{H}, \mathrm{H}+\mathrm{L}^{*}$, etc.).

\section{Study 2: An acceptability judgment task}

Twenty-five monolingual speakers of Castilian Spanish participated in this study. The experimental stimuli consist of a $3 \times 2$ design: Three discourse conditions - local antecedent, new referent, and wh-answer - and two syntactic conditions - utterances with fronted versus non-fronted constituents.

A linear mixed model with the maximal random structure (by-participant slope and intercept, by-item slope and intercept) converged when conducted in R-Studio. The inferential results show significant effects by Discourse $(F(2,26)$ $=17.79, \mathrm{p}<0.001)$ and Syntax $(\mathrm{F}(1,33)=7.14, \mathrm{p}<0.01)$, as well as a significant Discourse*Syntax interaction $(\mathrm{F}(2,18)=3.45, \mathrm{p}<0.05)$. Further, the Tuckey HSD pairwise comparisons show that CLLD is significantly dispreferred in the wh condition in comparison to the local $(\mathrm{p}<0.001, \mathrm{~g}=1.11)$ and new $(\mathrm{p}<0.02, \mathrm{~g}=0.67)$ conditions, with a non-significant different between these two $(\mathrm{p}=0.30, \mathrm{~g}=0.31)$. Further, there are no significant differences between CLLD and canonical utterances in the two conditions 
where CLLD is preferred; namely, the local $(\mathrm{p}=0.97, \mathrm{~g}=0.14)$ and new $(\mathrm{p}=$ $0.13, \mathrm{~g}=0.57)$ conditions.

\section{Study 3: A scripted production task}

Twenty-two monolingual speakers of Castilian Spanish participated in this study; twelve in Session 1 and ten in Session 2. The experimental stimuli consist of a $3 \times 2$ design: Three discourse conditions - contrastive-focus (CF), contrastive-topic (CT), given-topic (GT) - and two syntactic conditions fronted constituents in Session 1 versus non-fronted constituents in Session 2.

Table 1 presents the results from R-Studio, indicating the session, the variable, the analysis, the converging random structure, the intercept effect, and the three pairwise comparisons for each of the analyses.

Table 1: Inferential results of the scripted production task

\begin{tabular}{|l|l|l|l|l|l|l|l|}
\hline Session & Variable & Analysis & $\begin{array}{l}\text { Random } \\
\text { Structure }\end{array}$ & Sig.? & CF-CT & CF-GT & CT-GT \\
\hline 1 & Accent & GLMM & Intercepts & $*$ & n.s. & n.s. & n.s. \\
\hline 1 & Tone & GLMM & Intercepts & $*$ & $*$ & $*$ & n.s. \\
\hline 1 & Range & LMM & Maximal & $*$ & n.s. & $*$ & n.s. \\
\hline 2 & Accent & GLMM & Intercepts & n.s. & n.s. & n.s. & n.s. \\
\hline 2 & Tone & GLMM & Intercepts & $*$ & $*$ & $*$ & $*$ \\
\hline 2 & Range & LMM & Maximal & $*$ & n.s. & n.s. & n.s. \\
\hline
\end{tabular}

\section{Discussion}

I argue that the corpus results show that CLLD receives multiple discursive interpretations (RQ1). Second, the findings from the qualitative comparisons between CLLD and its canonical counterpart, further indicate that canonical utterances do fulfil the same discourse context as CLLD utterances do (RQ2). Third, the discourse-phonology results show that there is not a one-to-one correlation between the discourse function and the intonation of a fronted constituent (RQ3). Given the lack of actual instances of FF in the corpus, there is no evidence for or against the presence of a contrastive intonation for FF (RQ4).

For the acceptability results, first, the significant difference between whCLLD and the other two CLLD-types indicates that, while CLLD is acceptable in multiple discourse contexts (local antecedent and new referent), the interpretation of CLLD is not completely free (wh-answer) (RQ1). Further, the lack of significant differences between CLLD and its canonical counterparts in the local and new conditions indicate that canonical utterances do in fact fulfil the same discourse functions as CLLD does (RQ2). These findings provide further confirmation of the discourse-syntax findings from Study 1. 
For the production results, first, the lack of significant differences in the pairwise comparisons show that different discourse functions do not correlate with different pitch accents (RQ3); as in Study 1, Study 3 did not find a one-toone correlation between discourse and pitch accent. Second, contrastive-focus is significantly different than both topic-types for boundary tones in Session 1, which aligns with a positive answer to RQ4. The results for boundary in Session 2 and for range in both sessions do not indicate a clear pattern for a positive nor a negative answer to RQ4.

These findings have extensive implications. First, the flexibility in word order found in Studies 1 and 2, as well as the lack of correlation between discourse and pitch-accent in Studies 1 and 3, challenge Bianchi and Frascarelli's (2010) empirical predictions. Second, the fact CLLD is not accepted as an answer to a wh-word shows that this construction is not completely free from discourse restrictions, unlike predicted by Rubio Alcalá's (2014). Lastly, the fact that CLLD is accepted both when referring to a local antecedent as well as a to a newly introduced referent, challenges López's (2009) predictions.

Putting it all together, none of the three previous models on the discoursesyntax-phonology interface truly capture the empirical picture resulting from the three studies presented in this manuscript. Therefore, a new model of this 3-way interface is necessary. This task, however, is left for future inquiry (e.g., Sequeros-Valle, forthcoming) given the descriptive nature of the present paper.

\section{References}

Bianchi, V., Frascarelli, M. 2010. Is topic a root phenomenon? Iberia: An International Journal of Theoretical Linguistics, 2(1), 43-88.

Brunetti, L., Bott, S., Costa, J., Vallduví E. 2009. NOCANDO: A multilingual annotated corpus for the study of Information Structure. Presentation at the 5 th Corpus Linguistics Conference. The University of Liverpool.

Götze, M., Weskott, T., Endriss, C., Fiedler, I., Hinterwimmer, S., Petrova, S., Schwarz, A., Skopeteas, S., Stoel, R. 2007. Information structure. In S. Dipper, M. Götze, S. Skopeteas (Eds.), Information Structure in Cross-Linguistic Corpora (pp. 147-187). Potsdam: Interdisciplinary Studies of Information Structure.

López, L. 2009. A derivational syntax for information structure. Oxford, England: Oxford University Press. · Rubio Alcalá, C. (2014). Syntactic constraints of topicalization phenomena. Ph.D. dissertation, Universitat Autònoma de Barcelona.

Sequeros-Valle, J. (forthcoming). $[+\mathrm{a}],[+\mathrm{p}]$, and $[+\mathrm{c}]$ : A typology of discourse in Spanish. Typology of Morphosyntactic Parameters. 